\title{
The potential to quantify polypharmacy in older adult hospital inpatients using electronic prescribing software: a feasibility study
}

\author{
Nick Connor ${ }^{1} \cdot$ Simon Woodward ${ }^{1} \cdot$ Mark Norwood $^{1} \cdot$ Nigel Sturrock $^{1} \cdot$ James Woodard $^{1} \cdot$ Rob Skelly $^{1}$. \\ Richard Butterfield ${ }^{1} \cdot$ Sarah Lewis $^{2} \cdot$ Andrew Fogarty $^{2}$
}

Received: 20 December 2019 / Accepted: 10 March 2020 / Published online: 16 March 2020

(C) The Author(s) 2020

\begin{abstract}
Polypharmacy in older adults is a growing problem, as some drugs may be either unnecessary or even harmful. Admission to hospital under a Medicine for the Elderly specialist physicians represents an opportunity to review patients' medication. The recent introduction of electronic prescribing to some hospitals in the United Kingdom allows the development of tools to measure polypharmacy in in-patients, and subsequently to assess the efficacy of interventions that aim to optimize medication prescribing. We tested the feasibility of developing an Excel-based software code that measured the number of medications a group of patients were taking at admission and how many of these were still prescribed on discharge. Electronic prescribing data was obtained from the Royal Derby Hospital, over a period of 52 weeks from April 2017 to March 2018 for all patients over the age of 65 years who were admitted onto the medicine for the elderly wards and subsequently discharged. On admission, the median number of eligible medications was 11 (interquartile range IQR 8 to 15). At the time of discharge, the median number of eligible medications retained since admission was 9 (IQR 6 to 12). This represents a median number of medications that have been removed from the current medication regimen of 2 (IQR 1 to $3, p<0.001$ ). Electronic prescribing software in hospitals allows the development of tools to measure the burden of medications, and to examine the efficacy of future interventions that are developed to optimize drug prescribing in older adults.
\end{abstract}

Keywords Polypharmacy $\cdot$ Older adults $\cdot$ Electronic prescribing $\cdot$ Drugs

\section{Introduction}

The demographic profile of countries in Western Europe and Japan is changing as a consequence of longer life expectancy, which results in more elderly population living for longer, often with multiple co-morbidities [1]. Physicians caring for these patients recognize that as a consequence of these

Nick Connor and Simon Woodward contributed equally to this work.

Electronic supplementary material The online version of this article (https://doi.org/10.1007/s12553-020-00419-4) contains supplementary material, which is available to authorized users.

Andrew Fogarty

andrew.fogarty@nottingham.ac.uk

1 University Hospitals of Derby and Burton NHS Foundation Trust, Uttoxeter Rd, Derby DE22 3NE, UK

2 Division of Epidemiology and Public Health, NIHR Biomedical Research Centre, University of Nottingham, Nottingham City Hospital, Nottingham NG5 1PB, UK diseases, in conjunction often with multiple healthcare episodes with different clinicians, the total number of medications can increase to a level where some of the medications are of minimal benefit to the patient, or even counterproductive to their well-being as side-effects and interactions between medications occur in a relatively vulnerable population. The accumulation of multiple medications has been termed 'polypharmacy' [2] and contact with a physician with specialist expertise in the complexity of caring for older adults during an acute hospital admission represents a unique opportunity to discuss treatment priorities and review these medicines and ensure that the patient is only on those which are currently required.

The problem of polypharmacy has been recognized for decades in primary care, and this has led to a wide range of epidemiological and clinical studies based in this setting that aim to quantify and also address this issue (ref). These have been enabled by the availability of electronic patient databases in primary care that often include medications, which permits targeting specific medications and also quantifying the total 
individual burden of medication. Hospital doctors who care for the elderly are also very aware of the risks of polypharmacy in their patient population, but until very recently they have had to rely on individual clinical review of patients' medication as hospitals generally used paper drug records. However, as electronic prescribing software is now becoming available in UK (and other countries) hospitals, this allows the development of software tools that can quantify the scale of the problem of polypharmacy and the efficacy of interventions designed to address it at the institutional level for the first time.

The introduction of electronic prescribing software in secondary care is generally driven by an aim to improve the safety and efficiency of prescribing and delivering medications to patients. However, it also provides the opportunity to measure the burden of medications at the time of admission to hospital, and subsequently when the patient is discharged, thus allowing assessment of how this has changed during the course of the hospital episode. This is particularly important in the medical care of elderly patients, whose doctors are trained to use hospital admissions as an opportunity of review the appropriateness of all medications. We have used electronic prescribing software to quantify the polypharmacy burden, and how it has changed during the course of admission under the Medicine for the Elderly speciality wards at a busy UK teaching hospital as part of an initial feasibility study. This can subsequently be used to develop appropriate interventions that target polypharmacy in this population.

\section{Methodology}

\subsection{Study population}

Electronic prescribing data was obtained from the Royal Derby Hospital, a busy acute teaching hospital in the UK, over a period of 52 weeks from April 2017 to March 2018 of all patients over the age of 65 years who were admitted onto the specialist Medical Care for the Elderly wards and subsequently discharged from the hospital. This was a feasibility study to text the hypothesis that it was possible to quantify the burden of polypharmacy in an elderly in patient population using electronic prescribing software.

\subsection{Prescribing data}

The main outcome of interest was the number of drugs that a patient was prescribed: firstly, at the time of admission to hospital; and secondly, how many of these medications were prescribed at the time of discharge from hospital. The data were obtained from the prescribing software system (iCM, Isoft) for all eligible individuals.

\subsection{Generation of polypharmacy software tool}

Once the anonymized medication data for all eligible patients was downloaded from the prescribing software system (iCM, Isoft) for all eligible individuals at admission and discharge, these were imported into a SQL database. As a consequence of oral corticosteroids, antibiotics and nebulized medications sometimes being continued for a short period after discharge, these were excluded from all analyses, as these were likely to not reflect the longer term impact of the total burden of medications after discharge. The main data analysis was done using SQL, Excel, and Stata. The SQL code is provided as an appendix (Appendix) permitting replication of the data extraction and cleaning. In brief, the software provided a count of the number of medications at admission firstly, and how many of these medications were prescribed at the time of discharge from hospital after exclusion of oral corticosteroids, antibiotics and nebulized medications.

\subsection{Data analysis}

The admission medications were used to generate a count of the number of medications that a patient was admitted on. A similar approach was used for the number of medications that were retained until the time of discharge. These were used to generate summary statistics of the median number and range of the total number of medications of interest, and the differences between these two groups analysed using the Wilcoxon signed rank test (Stata statistical software). The validity of the electronic prescribing data was cross checked with medical and pharmacy staff to ensure the integrity of the data.

\section{Results}

Data were available on 3454 admission episodes. The median age of the study population was 86 years (interquartile range 80 to 91 ).

On admission, the median number of eligible medications was 11 (interquartile range IQR: 8 to 15 ), with the proportion of patients having been prescribed five or more and ten or more eligible medications being $94 \%$ and $64 \%$ respectively (Fig. 1). At the time of discharge, the median number of eligible medications retained since admission was 9 (IQR 6 to 12), with the proportion of patients having been prescribed five or more and ten or more eligible medications being $88 \%$ and $46 \%$ respectively (Fig. 1). This represents a median number of medications that have been removed from the current medication regimen of 2 (IQR 1 to $3, p<0.001$, Wilcoxon signed rank test). The distribution of the change in medications is presented in Fig. 2. 
Fig. 1 Number of drugs prescribed on admission and the number of these medications prescribed at time of discharge

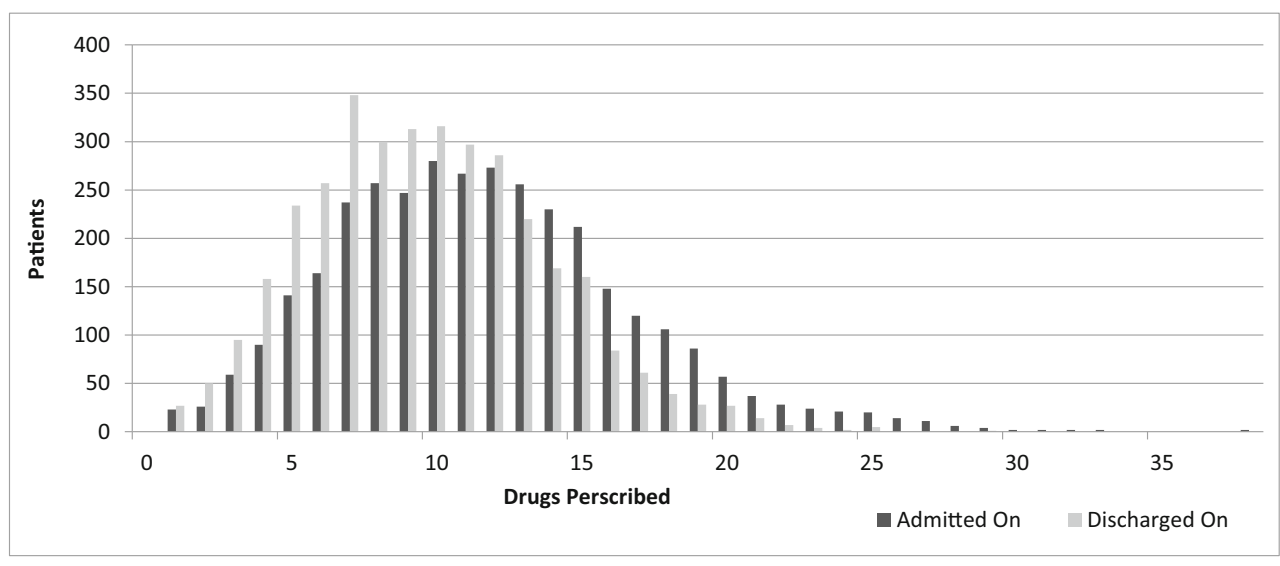

\section{Discussion}

We have used electronic prescribing system data describe the number of medications that elderly patients admitted to a busy acute teaching hospital are taking, and how this decreases after discharge from hospital under the care of a physician with a specialist interest in Medical Care of the Elderly. This software is relatively new to secondary care in the United Kingdom, and provides a novel tool that can help support clinicians caring for this patient group to audit trends in polypharmacy over time, assess effectiveness of interventions that aim to target polypharmacy, as well as facilitating the targeting of specific drugs that may be considered inappropriate for this population.

The strengths of the analysis include the confidence that we have captured data in $100 \%$ of eligible patient episodes as a consequence of the inclusiveness of the electronic prescribing software. This was a feasibility study designed to demonstrate the proof-of-concept that electronic prescribing systems can be used to measure the burden of polypharmacy in an elderly inpatient population. As a consequence, it was delivered in a single centre, and the data presented are not necessarily generalizable to other centres where patient demographics and medical practice may vary. The limitations are that it is essentially an epidemiological tool that can observe and describe the activity of the secondary care doctors, but does not permit correlation with the clinical condition that necessitates prescribing of medications, and hence cannot allow evaluation of how appropriate the prescribing of these medications is. This is inevitable, as the responsibility for prescribing is with the attending physician. It is important to acknowledge that the term 'polypharmacy' is descriptive, and not judgmental, and is used in this analysis as a summary statistic for the total medication burden. As we wished to demonstrate that electronic prescribing has the potential to quantify the number of medications, we excluded new medications that were started in hospital from the analysis. Hence, some of the observed decrease will be a consequence of the substitution of new medications for existing ones, as part of the clinical treatment. The current data and analysis demonstrate that it is feasible to measure change in polypharmacy burden in an elderly inpatient population using electronic prescribing software, and hence future analyses will aim to proceed to determine what are the changes in medication, are they evidence-based or not and can this approach be used to systematically introduce and assess institution-based interventions to rationalize patient medication and improve patient care? The software code is provided in the appendix in the SQL format as this is a commonly used database that can be used by any interested researchers, and adapted to local circumstances as necessary.
Fig. 2 Number of drugs prescribed prior to arrival but not on discharge

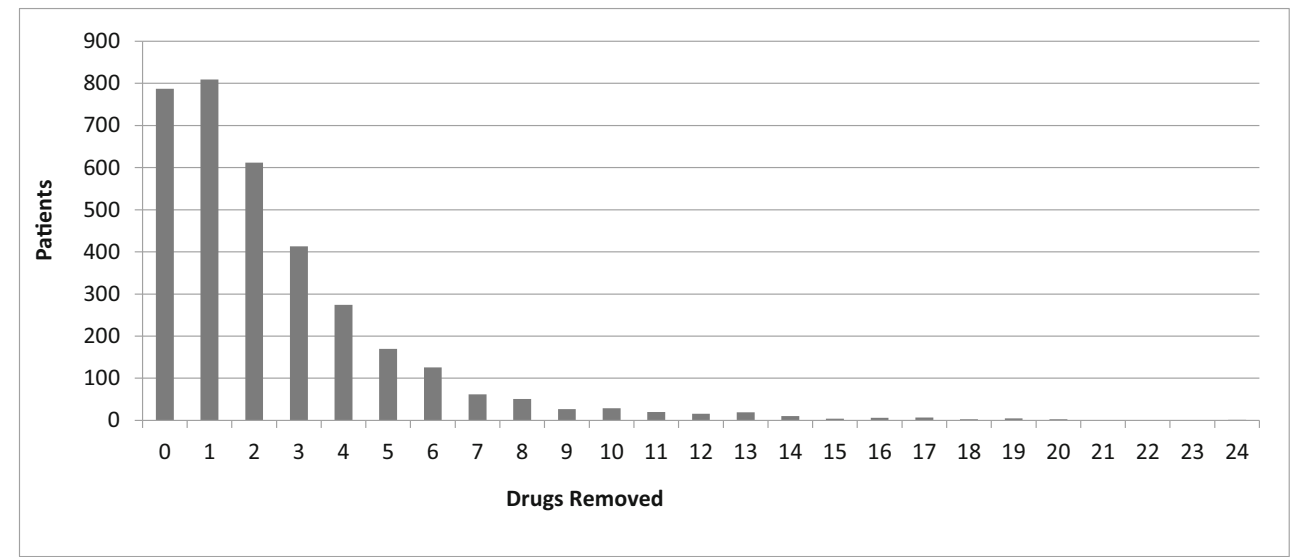


Polypharmacy is well recognized as a concern in caring for older adult patients [3], and there have been many initiatives to try and address this. These vary from targeting the education of doctors and associated healthcare professionals to increase awareness of these issues to drug reviews of the appropriateness of medications in elderly populations by pharmacists [4]. Although polypharmacy has been described previously using electronic primary care prescribing databases [1], this approach has not been systemically used in secondary care in the UK. The observation that the median number of medications decreases by two drugs during the course of a medical admission demonstrates that Medical Care of the Elderly physicians are effective at reviewing medications, and the scale of the decrease is plausible and consistent with clinical expectations.

The implication of this analysis is that electronic prescribing software can be used to develop a tool to audit efficacy of decreasing polypharmacy in an elderly population of secondary care patients. We have added the SQL code to this manuscript as an appendix, so that interested individuals with access to comparable electronic prescribing software data can use this tool freely. In the future it could be used to initiate a review of drugs which may be causing risks to patients' wellbeing. One example would be quinine, which has been commonly used in the past as a symptomatic treatment for leg cramps, but has also been associated with increased mortality rates in more recent randomized controlled trials [5]. Ultimately, it would be optimal to work towards electronic prescribing software that covers both primary and secondary heathcare sectors, as this would provide a longitudinal perspective of temporal changes in medication burden in the elderly, and how primary and secondary care interact to modify this.

\section{Conclusion}

In conclusion, electronic prescribing systems can be used to quantify polypharmacy in an elderly population, and to assess the overall efficacy of specialist physicians caring for older adult patients to address this issue during admission to hospital. Our software code is available in the Appendix for institutions that wish to replicate and build on our work. Over time, this approach has the potential to provide an epidemiological tool to help support doctors who care for older adults in the context of increasing demand.

Funding information The Health Foundation Advancing Applied Analytics Programme.

\section{Compliance with ethical standards}

Conflict of interest The authors declare that they have no conflict of interest.

Ethical approval The study was an evaluation of the development of a health service development and was registered as an audit using anonymised data.

Open Access This article is licensed under a Creative Commons Attribution 4.0 International License, which permits use, sharing, adaptation, distribution and reproduction in any medium or format, as long as you give appropriate credit to the original author(s) and the source, provide a link to the Creative Commons licence, and indicate if changes were made. The images or other third party material in this article are included in the article's Creative Commons licence, unless indicated otherwise in a credit line to the material. If material is not included in the article's Creative Commons licence and your intended use is not permitted by statutory regulation or exceeds the permitted use, you will need to obtain permission directly from the copyright holder. To view a copy of this licence, visit http://creativecommons.org/licenses/by/4.0/.

\section{References}

1. Duerden M, Avery T, Payne R. Polypharmacy and medicines optimisation. Making it safe and sound. The Kings Fund. 2013: https:// www.kingsfund.org.uk/sites/default/files/field/field_publication file/polypharmacy-and-medicines-optimisation-kingsfund-nov13. pdf. Accessed 1/3/2019.

2. Masnoon N, Shakib S, Kalisch-Ellett L, Caughey G. What is polypharmacy? A systematic review of definitions. BMC Geriatr. 2017;17:230. https://doi.org/10.1186/s12877-017-0621-2.

3. Spinewine A, Schmader K, Barber N, Hughes C, Lapane K, Swine $\mathrm{C}$, et al. Appropriate prescribing in elderly people: how well can it be measured and optimised? Lancet. 2007;370:173-84.

4. Payne R, Avery A. Polypharmacy: One of the greatest prescribing challenges in general practice. Brit J General Practice. 2011:83-4.

5. Fardet L, Nazareth I, Petersen I. Association between long-term quinine exposure and all-cause mortality. JAMA. 2017;317:1907-9.

Publisher's note Springer Nature remains neutral with regard to jurisdictional claims in published maps and institutional affiliations. 